The INL is a

U.S. Department of Energy

National Laboratory

operated by

Battelle Energy Alliance

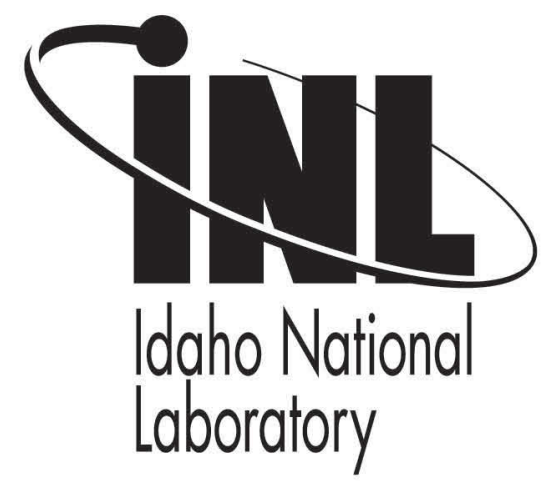

INL/CON-12-26938

PREPRINT

\section{The Mars Hopper:}

Development, Simulation

and Experimental

Validation of a

Radioisotope Exploration

Probe for the Martian

Surface

\section{AIAA Space 2012}

Nathan D. Jerred

Spencer Cooley

Robert C. O'Brien

Steven D. Howe

James E. O'Brien

\section{September 2012}

This is a preprint of a paper intended for publication in a journal or proceedings. Since changes may be made before publication, this preprint should not be cited or reproduced without permission of the author. This document was prepared as an account of work sponsored by an agency of the United States Government. Neither the United States Government nor any agency thereof, or any of their employees, makes any warranty, expressed or implied, or assumes any legal liability or responsibility for any third party's use, or the results of such use, of any information, apparatus, product or process disclosed in this report, or represents that its use by such third party would not infringe privately owned rights. The views expressed in this paper are not necessarily those of the United States Government or the sponsoring agency. 


\title{
The Mars Hopper: Development, Simulation and Experimental Validation of a Radioisotope Exploration Probe for the Martian Surface
}

\author{
Nathan D. Jerred ${ }^{1}$, Spencer Cooley $^{2}$, Robert C. O'Brien ${ }^{3}$ and Steven D. Howe ${ }^{4}$ \\ Center for Space Nuclear Research, Idaho Falls, Idaho, 83401 \\ and \\ James E. O’Brien ${ }^{5}$ \\ Idaho National Laboratory, Idaho Falls, Idaho, 83401
}

\begin{abstract}
An advanced exploration probe has been proposed by the Center for Space Nuclear Research (CSNR) to acquire detailed data from the Martian surface and subsurface, 'hop' large distances to multiple sites in short periods of time and have the ability to perform this task repeatedly. Although several similar flying vehicles have been proposed utilizing various power sources and complex designs, e.g. solar-electric and chemical-based, the CSNR's Mars Hopper is based on a radioisotope thermal rocket (RTR) concept. The Mars Hopper's design relies on the high specific energies $[\mathrm{J} / \mathrm{kg}]$ of radioisotopes and enhances their low specific power $[\mathrm{W} / \mathrm{kg}]$ through the use of a thermal capacitance material to store thermal energy over time. During operation, the RTR transfers the stored thermal energy to a flowing gas, which is then expanded through a converging-diverging nozzle, producing thrust. Between flights, the platform will have ample time to perform in-depth science at each location while the propellant tanks and thermal capacitor recharge. Recharging the propellant tanks is accomplished by sublimation freezing of the ambient $\mathrm{CO}_{2}$ atmosphere with a cryocooler, followed by heating and pressurization to yield a liquid storage state. The proposed Mars Hopper will undergo a ballistic flight, consuming the propellant in both ascent and descent, and by using multiple hopper platforms, information can be gathered on a global scale, enabling better resource resolution and providing valuable information for a possible Mars sample-return mission. The CSNR, collaborating with the Idaho National Laboratory (INL) and three universities (University of Idaho, Utah State University and Oregon State University), has identified key components and sub-systems necessary for the proposed hopper. Current project activities include the development of a lab-scale prototypic Mars Hopper and test facility, along with computational fluid dynamics (CFD)/thermal-hydraulic models to yield a better understanding of the heat transfer process and complex nature of turbulent $\mathrm{CO}_{2}$ flow. Laboratory experimentation will aid design iterations and the development of both tethered and free-flying terrestrial hoppers that utilize an electrically heated core. The knowledge base acquired from these activities will refine the Mars Hopper's future performance and optimize the RTR core components prior to constructing the final design.
\end{abstract}

\footnotetext{
${ }^{1}$ Research Scientist, Center for Space Nuclear Research, 995 University Blvd.

${ }^{2}$ Next-Degree Student, Center for Space Nuclear Research, 995 University Blvd.

${ }^{3}$ Research Scientist, Center for Space Nuclear Research, 995 University Blvd.

${ }^{4}$ Center Director, Center for Space Nuclear Research, 995 University Blvd.

${ }^{5}$ Senior Research Engineer, Experimental Programs, 2525 Fremont Ave MS 3870.
} 


\section{Introduction}

$\mathrm{T}$ HE requirements for performance by planetary exploration missions are increasing. Landing at a single location to take data is no longer sufficient and due to the increasing cost, the missions that provide mobile platforms that can acquire data at displaced locations are becoming more attractive. The Mars Exploration Rovers, Spirit and Opportunity, have performed remarkably for 7 years and have covered 4.8 and 21.6 miles, respectively. The Phoenix lander has sampled the Martian surface close to the northern polar cap of Mars but only at one location. Spot sampling at a few locations, especially locations that are safe landing sites, will not provide an accurate geophysical map of Mars. In addition, if humans are ever going to land on Mars, we must produce a much more detailed map of the resources, terrain and subsurface in order to determine an optimal landing site.

Robotic probes for planetary exploration are an excellent way to acquire high quality data to validate orbiting observations. The orbiting systems allow large area coverage and the ground systems provide spot validation. The ability to fly such missions is becoming ever more costly and increasingly dependent on international collaboration in order to maintain an adequate mission frequency. In addition extended lifetime, long-range exploration and sampling to significant depths of several meters requires significantly more power than what can be provided from solar cells. Furthermore, the unavailability of ${ }^{238} \mathrm{Pu}$ for nuclear batteries means that extended lifetime and long-range exploration will become more difficult. According to the report from the Radioisotope Power Systems Committee of the National Research Council, "Radioisotope Power Systems: An Imperative for Maintaining U.S. Leadership in Space Exploration", ${ }^{238} \mathrm{Pu}$ may not be available after the last Flagship-scale mission to Europa in 2020.

More ambitious technologies and scientific methods must be developed to increase the science return for each launch, thus increasing the scientific value for the money spent for each mission. Several previous studies have proposed the use of "hoppers" powered by various means. ${ }^{1-3}$ However, these concepts suffered from both short range and relatively short operational durations. Conceivably, if an instrumented platform could be placed on the surface of a planet, highly detailed data could be acquired. This data would include both surface and subsurface findings. This platform would also be able to travel large distances to multiple sites. In addition, data collection could be repeated through many iterations from one site to the next, allowing for accurate mapping and sampling of the entire planetary surface. A higher resolution would be provided than that of orbiting platforms and the range would far extend those of current in-situ missions. In addition, if several such platforms could be simultaneously deployed from a single launch vehicle, a surface network of science stations would be possible that provide long term assessment of meteorological conditions.

The Center for Space Nuclear Research (CSNR) is designing an instrumented platform that can acquire detailed data at hundreds of locations during its lifetime - a Mars Hopper. The platform will be able to "hop" from one location to the next every 5-7 days with a distance ranging from 5-10 km per hop. Each platform will weigh around $52 \mathrm{kgs}$ dry (i.e. empty of propellant), which is the condition at deployment. Consequently, several platforms may be deployed on a single launch from Earth, and with a lifetime estimate of 5-7 years, the entire surface of Mars could be mapped in detail by several platforms. Furthermore, the basic platform can be deployed to Europa, Titan, and even Venus with minor alterations - similar propulsion system with essentially the same operations.

\section{Concept}

The basis of the concept is to utilize decay heat from a radioactive isotope to heat the core material to high temperatures, diverting some thermal power to operate a cryocooler. The cryocooler sublimate freezes the Martian atmosphere and heats it to a liquid state, storing it in a separate propellant tank at roughly 2.8 MPa. After the peak temperature in the core is reached $\mathrm{CO}_{2}$ is injected into the core, where thermal energy is transferred to the flowing gas which is expanded through a nozzle, and allowed to produce thrust. Half of the propellant is consumed through the ascent phase and after a ballistic coast, the remaining propellant is used for a soft landing. Once landed, samples are collected and the process repeats.

The Mars Hopper concept utilizes energy from radioisotopic decay in a manner different from any existing radioisotope power sources - as a thermal capacitor. Radioisotope sources have very high specific energy [J/kg] while having rather low specific power $[\mathrm{W} / \mathrm{kg}$ ]. Therefore, if thermal energy from radioisotopic decay can be stored over long periods of time and if that power can be dramatically increased through short periods of time, then a radioisotope thermal rocket (RTR) can be possible.

The most optimal radioisotope choice to provide the needed energy for operation of an RTR is ${ }^{238} \mathrm{Pu}$. This plutonium isotope has a long history of use in nuclear batteries and is more manageable than other possible radioisotope choices. However, ${ }^{238} \mathrm{Pu}$ stocks are dwindling, but with minimal engine changes, several other radioisotopes could be used as substitutes. Radioisotope power is extremely advantageous and its benefits, when compared to other power sources, should be noted. For example, ${ }^{238} \mathrm{Pu}$ has a specific energy of $1.6 \times 10^{6} \mathrm{MJ} / \mathrm{kg}$ 
$\left(4.4 \times 10^{5} \mathrm{~kW}-\mathrm{hrs} / \mathrm{kg}\right)$. Factoring in a $6 \%$ conversion to electricity, NASA's radioisotope thermoelectric generators (RTGs) have $9.6 \times 10^{4} \mathrm{MJ} / \mathrm{kg}$ of electrical energy compared to $0.72 \mathrm{MJ} / \mathrm{kg}$ for lithium ion batteries. ${ }^{4,5}$ Additionally, radioisotopes provide longer mission lifetimes, with minimal redundancy needed in the power source and are the likely choice for missions to the outer planets where solar fluxes may be inadequate.

Integration of the RTR core and the radioisotope fuel goes beyond the traditional General Purpose Heat Source (GPHS) bricks used in an RTG, and instead focuses on encapsulating radioisotope fuel kernels (about $100 \mu \mathrm{m}$ in diameter) within a solid tungsten or tungsten-rhenium matrix. Recent developments in the fabrication of tungstenrhenium (W-Re) materials at the CSNR indicate that a solid, resilient, high-temperature matrix can be formed to encapsulate the radioisotope kernels. ${ }^{6,7}$ This encapsulation process will prevent the dispersion of the radioisotopes when exposed to the destructive forces associated with spacecraft re-entry during plausible launch abort scenarios or planetary impacts (in the case of failed in-situ probe deployment). The unique fabrication technique of the Spark Plasma Sintering (SPS) process being utilized at the CSNR allows for flexibility in the design of the final fuel form with minimal procedural changes. Figure 1 shows examples of a tungsten-based compact created at the CSNR, modeled after NERVA-era NTR fuel elements.

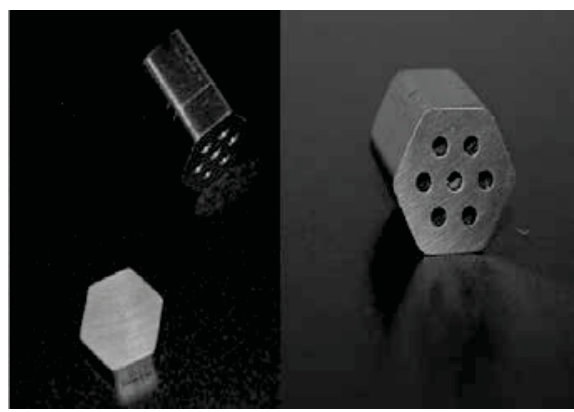

Figure 1. Tungsten elements fabricated in the SPS furnace reminiscent of the NERVA nuclear thermal rocket fuel elements. Samples were loaded with $\mathrm{CeO}_{2}(40 \%$ vol.), which acts as the fuel simulant. $^{6}$

To increase the specific power of an exploration probe, making the RTR feasible, the core must be comprised of a material exhibiting high specific heat, high density and high thermal conductivity with a relatively high melting temperature. Figure 2 shows several candidate materials expressing their specific heat $[\mathrm{J} / \mathrm{kg} * \mathrm{~K}]$ versus temperature $[\mathrm{K}]$.

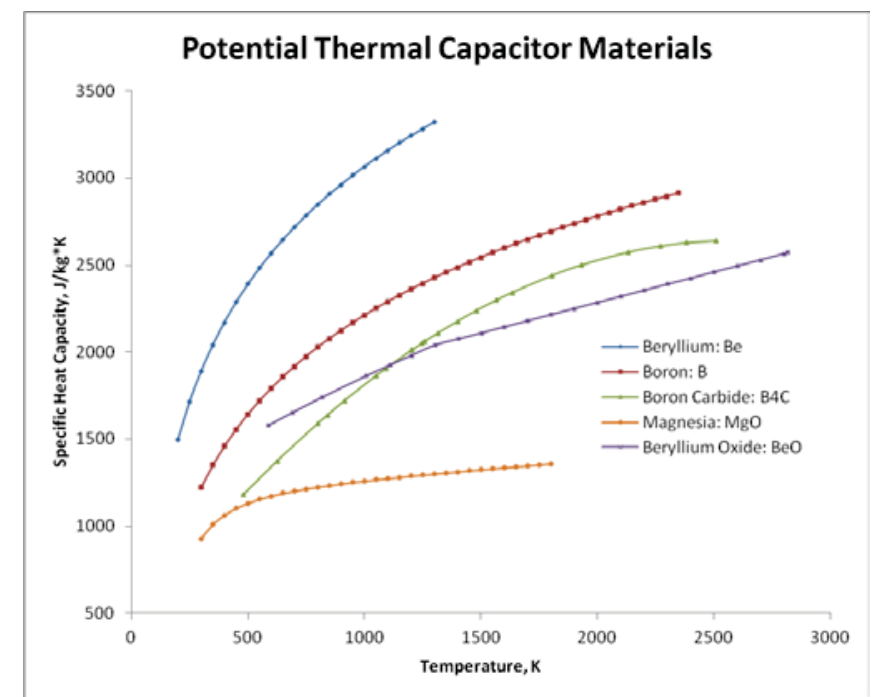

Figure 2. Specific heat capacity data of various materials as a function of temperature..$^{8-13}$ 
For this version of the Mars Hopper, beryllium was determined to be the most promising candidate with the potential to store $2.01 \mathrm{MJ} / \mathrm{kg}$ over a $500 \mathrm{~K}-1200 \mathrm{~K}$ temperature range. Although beryllium has the lowest melting temperature $\left(\mathrm{T}_{\text {melt }}=1500 \mathrm{~K}\right)$, an operational peak temperature of $1200 \mathrm{~K}$ becomes more manageable from a material selection and thermal cycling perspective, which can lead to higher confidence in the probe's operational lifetime. A very important aspect of the Mars Hopper is its design to utilize the $\mathrm{CO}_{2}$-rich atmosphere of Mars as propellant, limiting launch mass and increasing the probe's potential mission lifetimes. Two key subsystems will work in unison to perform the task of acquiring propellant. The first system is the energy conversion system, which will be designed to convert thermal energy from the core into electrical energy. This electric energy will then operate the propellant liquefaction subsystem through an integrated cryocooler. Several energy conversion systems exist, such as Stirling- or Brayton-based dynamic heat cycles, however to increase probe longevity a solid-state conversion system is the preferred choice. Historically, NASA has had great success with thermoelectric conversion systems, but they are inefficient and require a large isotopic loading, which is unlikely for ${ }^{238} \mathrm{Pu}$. Instead the hopper is being designed to operate using a thermal photovoltaic (TPV) energy conversion system, which has recently achieved higher conversion efficiencies. An insulation scheme can be designed so the TPV cells will utilize radiation losses from the core to produce the needed electrical power. Figure 3 shows a schematic of a TPV system.

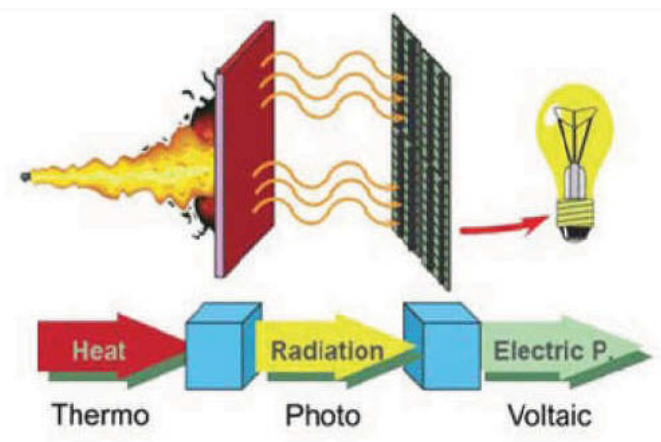

Figure 3. Schematic of a thermal photo-voltaic energy conversion system. ${ }^{14}$

The outer peripheries of the core can be made to further enhance the TPV conversion efficiency by incorporating a 2D photonic crystal structure upon a special emitter sheath. This specially designed sheath can be tailored to emit thermal radiation within a narrowed wavelength range to match the band-gap of the photo-voltaic cells being used. ${ }^{14}$

Preliminary studies have shown a cryocooler can be designed to operate on $240 \mathrm{~W}_{\mathrm{e}}$, acquiring the needed mass of propellant in seven to eight days. Additionally, while the liquefaction subsystem is operating the probe's scientific platform can operate simultaneously, gathering samples and performing experiments in the probe's general vicinity while waiting for adequate propellant to be collected. The liquefaction subsystem works by initially allowing the $\mathrm{CO}_{2}$ to sublimate freeze on to an internal cold finger. With the chamber closed, the solid $\mathrm{CO}_{2}$ will be slightly heated to a liquefied state and stored in an attached propellant tank. Although fractions of a gram of $\mathrm{CO}_{2}$ can be produced at any one time, continuous operation will produce the needed $25 \mathrm{~kg}$ of propellant for each hopping maneuver. Table 1 shows a summarized breakdown of the probe's potential performance. A more detailed assessment of the Mars Hopper can also be found in Steven D. Howe et al.

Table 1. Summarized performance data of the Mars Hopper. ${ }^{15}$

Total Stored Energy [J]

Isotope Thermal Power [W]

Core Max Temperature [K]

${ }^{238} \mathrm{PuO}_{2}$ Mass [kg]

Beryllium Mass [kg]

Designed Payload Mass [kg]

Propellant Mass [kg]

Total Dry Mass [kg]

Total Wet Mass [kg]

Total Burn Time [s]

Range [km]

$\begin{array}{ll}\rightarrow & 1.48 \times 10^{7} \\ \rightarrow & 1000 \\ \rightarrow & 1200 \\ \rightarrow & 2.5 \\ \rightarrow & 6.068 \\ \rightarrow & 10 \\ \rightarrow & 25 \\ \rightarrow & 50.744 \\ \rightarrow & 75.744 \\ \rightarrow & 22.86 \\ \rightarrow & 6.06\end{array}$




\section{Laboratory-scale Prototype and Simulation Development}

Early on in development of the Mars Hopper it was important to perform computational fluid dynamic (CFD) analysis of propellant flow and thermal hydraulic studies of the probe to overcome thermal isolation issues and to more accurately predict the actual performance of the hopper. However, due to the non-ideal-gas behavior of $\mathrm{CO}_{2}$, and to test various insulation schemes, the importance of designing a laboratory-scale test rig of the Mars Hopper was recognized in order to provide benchmark data for comparison to the simulations. With empirical data both CFD and thermal hydraulic codes can be validated, allowing for design iterations to be performed numerically in order to fine tune the probe's design and ultimately maximize its performance.

\section{A. Simulation Analysis}

Initially, simulation analysis was performed on the Mars Hopper probe using Star-CCM+ from CD-adapco. Attention was then shifted to performing analysis on the test rig using both the multiphysics code COMSOL and Star-CCM+ in order to benchmark simulation results. A computer-aided design (CAD) model of the test rig was loaded into Star CCM+ for CFD analysis of $\mathrm{CO}_{2}$ flow through the flow channels. Figure 4 shows preliminary results of $\mathrm{CO}_{2}$ gas flow through the test rig's core, heated to $1200 \mathrm{~K}$. As can be seen, gas temperatures are increased to about $1200 \mathrm{~K}$, with a slight drop in gas temperature occurring at the exiting plenum; likely due to the volume expansion that occurs at the plenum. One important design difference between the test rig and the Mars Hopper probe is the absence of a nozzle, which can be seen in the simulation. Instead, the test rig design incorporates two gas plenums, one for gas injection, and the other to handle the gas exhaust. The test rig design will be discussed in more detail below.

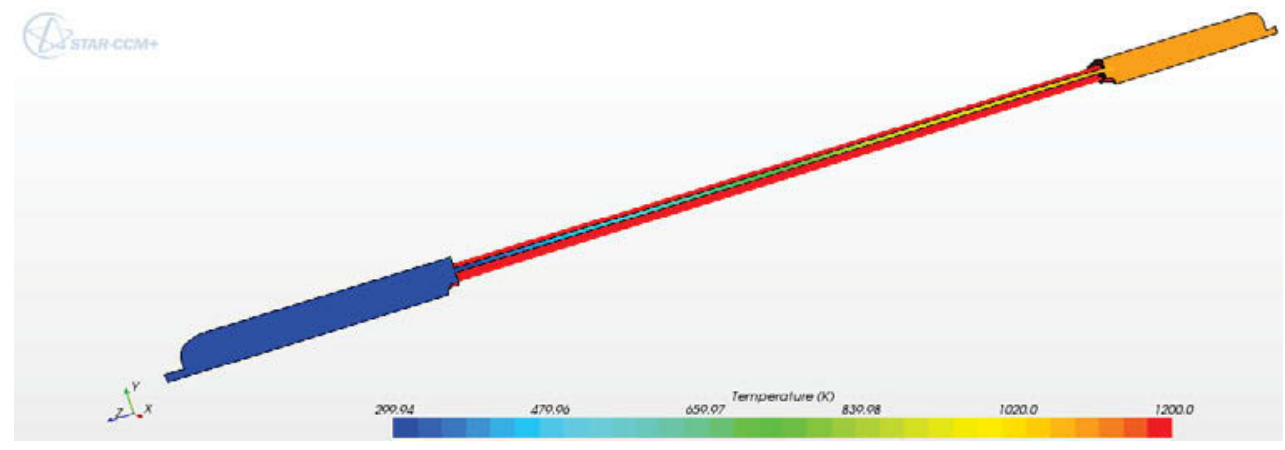

Figure 4. CFD analysis of the test rig using Star-CCM+. ${ }^{16}$

As mentioned above, a preliminary CFD analysis of gas flow through the test rig core indicated the gas flow to be turbulent, making the flow analysis of $\mathrm{CO}_{2}$ through the core to be complex. This resulted in more attention to be focused on the fabrication of the test rig and the encompassing test facility to house blow-down tests of the prototype before further simulations would be investigated.

\section{B. Laboratory-scale Prototype}

Through the current CSNR Mars Hopper task, a laboratory-scale prototype has been developed and fabricated, with the intent to gain a better understanding of heated $\mathrm{CO}_{2}$ gas flow, as well as aiding in the optimization of the Mars Hopper's overall performance. Although the initial test rig development is focused on the production of empirical data, it was also designed for the potential to perform both tethered and untethered flights in the future. The test rig will utilize two gas plenums for both gas injection and exhaust handling. The entire inner assembly is encased by a titanium shell which contains both the radial insulation scheme and the inner core. At the center of the core is a $275 \mathrm{~W}$ electric heater to simulate radioisotopic heat. To thermally isolate the core axially from the injection and exhaust plenums, a zirconia disk will be used. Tie rods connected to flanges fitted to each plenum will be used to axially compress the entire test rig through thermal cycling and blow-down testing.

Through initial designs of the test rig's inner core, the idea of using bundled rods, reminiscent of NTR hexagonal-based cores, was determined to be best suited from both a fabrication and materials handling perspective. As previously mentioned, an important aspect of the RTR core's performance is the incorporation of beryllium to act as the thermal capacitor to store energy. However, the use of bare beryllium can be hazardous. Therefore a metallic coating was applied to the beryllium to ease handling and blow-down testing concerns. In order to ensure the inner surfaces of the flow channels were properly coated through their entire length, the bundled rod core design 
was the most practical. This design allows for each rod to incorporate part of the flow channels on their outer perimeter. Therefore once the rods are bundled together a flow channel pattern is visible. For the metallic coating, a material exhibiting a similar coefficient of thermal expansion (CTE) to that of beryllium must be used to prevent flaking through thermal cycling, thus the nickel-based alloy Hastelloy C-276 was chosen. Hastelloy C-276 has a manufacturer reported CTE of $12.2 \mu \mathrm{m} / \mathrm{m}^{* \circ} \mathrm{C}$, which compliments that of beryllium's CTE.

Two rod designs were pursued, which produce two unique inner core assemblies and flow channel patterns. Figure 5 shows both inner assembly cross-sectional profiles where Fig. 5 (a) is made up of circular-based rods and Fig. 5 (b) of a hexagonal-based design. At the center of the inner assemblies is the heater rod (shown in red), which is encased in a Hastelloy C-276 shell designed to fit in the respective assembly. In order to radially contain the inner bundled rods, a Hastelloy C-276 shell is again employed for each respective design as seen in Fig. 5 (c).

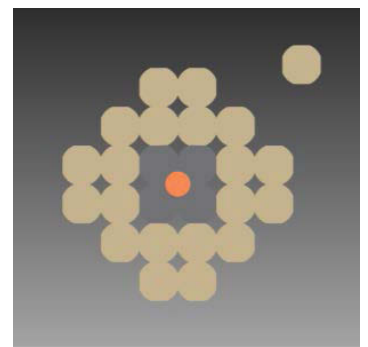

(a)

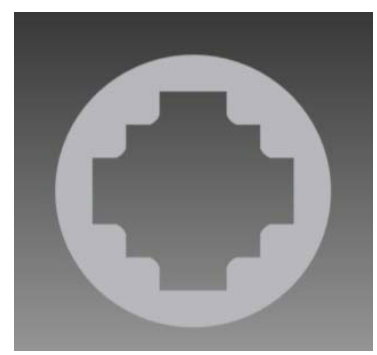

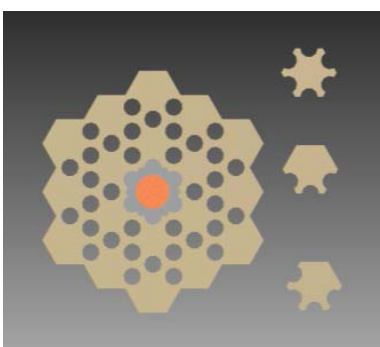

(b)

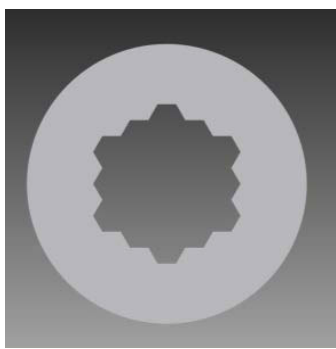

(c)

Figure 5. CAD drawings of the inner core assembly parts for the test rig where (a) shows the circularbased design, (b) shows the hexagonal-based design and (c) shows the outer shell for each bundled core design. ${ }^{17}$

Based off of initial rod designs, as seen in Fig. 5 (a) and (b), beryllium rods have been procured for the project. However, in order to perform testing at the available facilities, a set of rods for each design made of Hastelloy C-276 was fabricated as a surrogate for the beryllium rods. With the beryllium rods stored at Los Alamos' beryllium lab the current project's work scope will include thermal cycling the prototype to test its structural integrity and to perform preliminary blow-down testing with $\mathrm{CO}_{2}$ gas to better understand the test rig's performance and finalize its overall design. After these initial tests, final tests will be performed using the beryllium rods. Figure 6 shows a CAD drawing of a cross-sectional view of the test rig fully assembled.

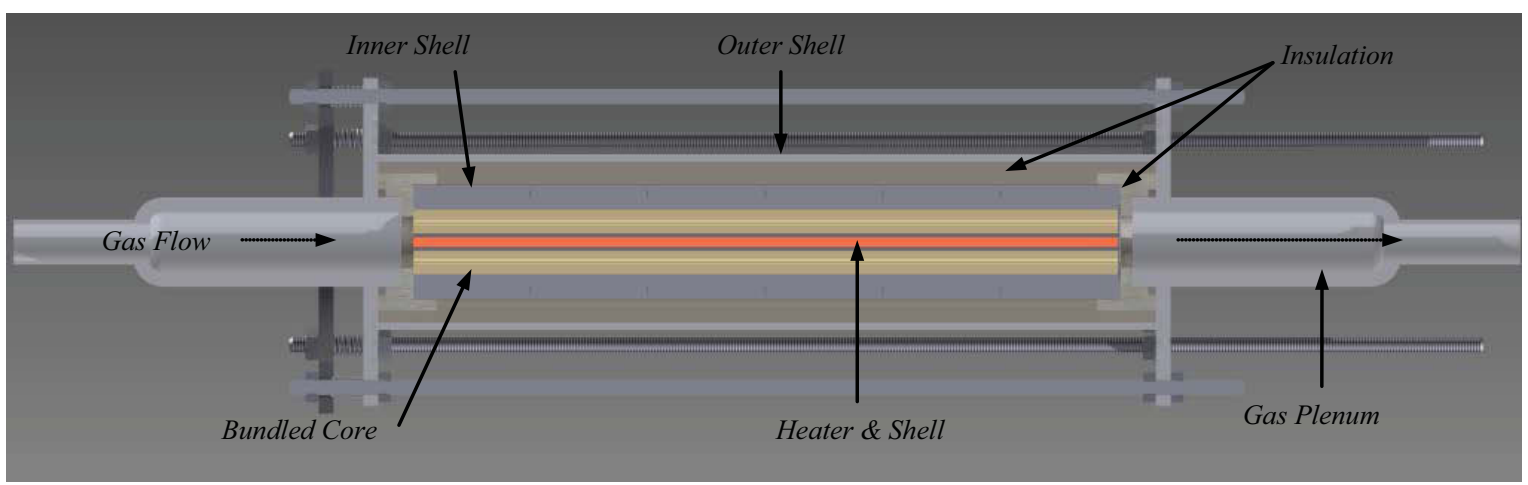

Figure 6. CAD drawing showing a cross-section view of the fully assembled test rig. ${ }^{17}$ 


\section{Mars Hopper Test Facility}

The test facility is primarily comprised of a pressure vessel to hold the hopper prototype for testing, which can be seen pictured in Fig. 7 (a) and (b). The pressure vessel will simulate Martian ambient conditions such as providing a rough vacuum of $0.01 \mathrm{~atm}$ with a $\mathrm{CO}_{2}$ atmosphere. Additionally, the vessel will also act as an engineered safety control in the event of a failed blow-down test. A pressurized train will be integrated with the test rig to safely inject as well as handle the hot $\mathrm{CO}_{2}$ gas.

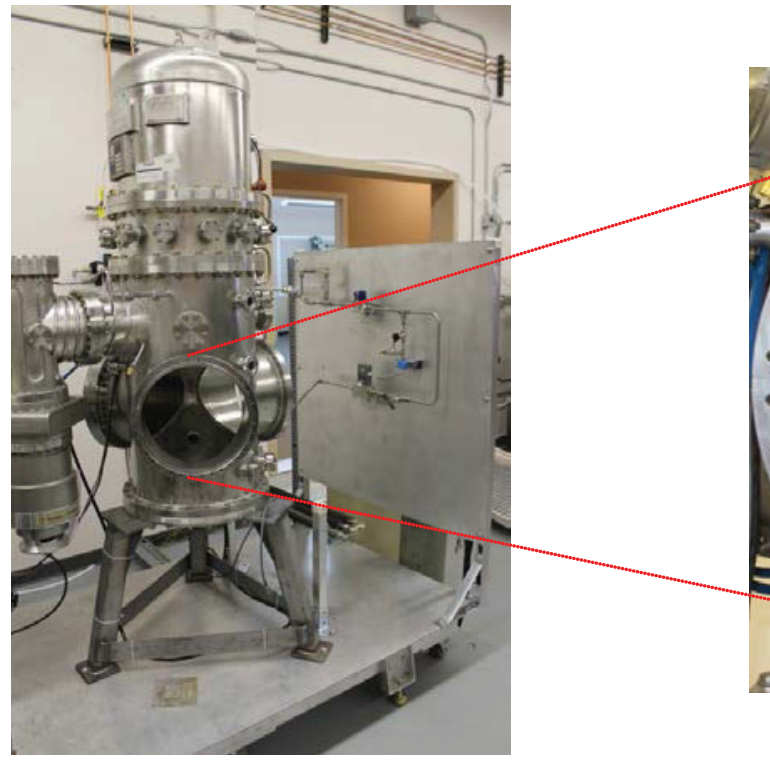

(a)

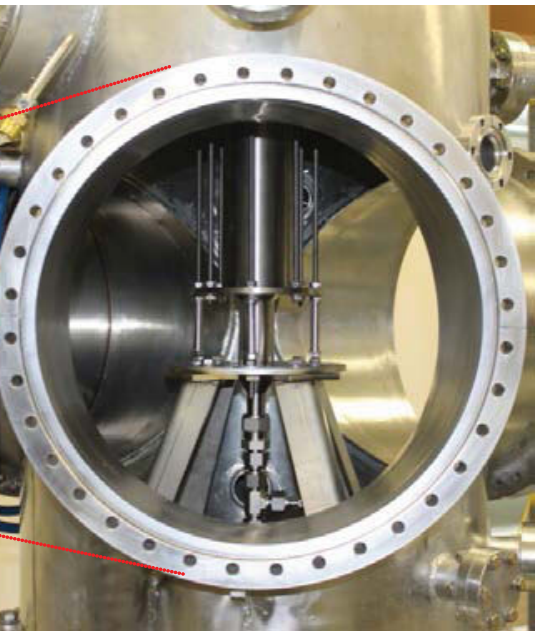

(b)

Figure 7. Shows portions of the test facility where (a) is the pressure vessel and (b) is the test rig assembled within the pressure vessel.

Upstream of the test rig and connected to the pressurized train is a mass flow meter and variable area flow valve to control and monitor gas flow rates through the prototype. Hot gas exiting the test rig will be passed through a finned heat exchanger and allowed to flow in to the general laboratory's exhaust system. The test rig itself will be fitted with various thermocouples in order to monitor the inner core temperature at varying axial positions, provide a control feedback loop to a PID controller for the heater and to monitor the outer shell temperature throughout each experiment. Pressure data of the gas will be gathered via an upstream pressure transducer, as well as a differential pressure transducer, measuring the pressure drop across the core. Additionally, gas temperatures will be measured upstream and downstream of the heated rig. The pressure vessel itself is a double-walled design with constant wall cooling provided by a continuous flow of water. To ensure inner wall temperature limits of the vessel are not exceeded, a thermocouple will also be attached directly adjacent to the test rig. A complete Labview suite will be used to monitor and record all temperature, pressure and gas flow rate data throughout the entire blow-down testing.

\section{Conclusion}

With decreasing budgets and the push to perform cheap science, the proposed Mars Hopper may be attractive due to its small size and simple design. Extending on the legacy of the current rover program, the use of multiple transient platforms can increase our knowledge base of the Martian planet and yield resource information on a global scale. With the ability to gather samples globally over several years a Mars sample return mission may prove to be viable.

This year's project efforts yielded two fabricated test rig designs and the assembly of a test facility to perform various tasks. With the tooling efforts coming to an end, preliminary thermal cycling and blow-down tests will be accomplished prior to the projects Sept. $30^{\text {th }}$ deadline. Future work will focus on substituting a mass flow controller for the current mass flow meter in order to provide a range of $\mathrm{CO}_{2}$ flow rates to the test rig. With data gathered from upcoming blow-down testing, CFD simulations will be able to be empirically validated and numerical optimization of the Mars Hopper can be performed. Additional future work will include pursuing the use of beryllium rods as the inner core material, as well as testing a proof of concept by performing both tethered and free-flier flight tests. 


\section{Acknowledgments}

The authors would like to thank Lisa Moore-McAteer and Eric Larsen, both of the Idaho National Laboratory, for their assistance in setting up the Mars Hopper test facility and its subsequent commissioning. Acknowledgment should be made for various hardware contributions made to support the assembly of the test facility by Keith Condie, Eric Larsen and Jim O'Brien. The authors would also like to acknowledge work that was conducted by peers from the CSNR Summer Fellowship Program as well as by senior design teams at Utah State University, University of Idaho and Oregon State University over previous years. Project funding was provided through INL's laboratory-directed research and development (LDRD) program via LDRD \#10-081.

\section{References}

${ }^{1}$ Shafirovich E., Salomon M., Gökalp I., “Mars hopper versus Mars rover”, Acta Astronautica, 2006, 59, 710 - 716.

${ }^{2}$ Montminy S., Dupuis S. and Champliaud H., Mechanical design of a hopper robot for planetary exploration using SMA as a unique source of power. Acta Astronautica, 2008. 62, 438-452.

${ }^{3}$ Fiorini P. and Burdeck J., Minimalist jumping robots for celestial exploration, The International Journal of Robotics Research, 2003, 22, 653-674.

${ }^{4}$ Lange R.G, Carroll W.P, Review of recent advances of radioisotope power systems, Energy Conversion \& Management, 2008, 49, 393-401.

${ }^{5}$ Tritt T. M. , Subramanian M. A., Thermoelectric Materials, Phenomena, and Applications: A Bird's Eye View, MRS Bulletin, 2006, 31, 188-194.

${ }^{6}$ O'Brien R. C., Ambrosi R. M., Bannister, N. P., et al., Spark Plasma Sintering of simulated radioisotope materials within tungsten cermets, Journal of Nuclear Materials, 2009, 393(1), 108-113.

${ }^{7}$ O’Brien R. C., Ambrosi R. M., Bannister, N. P., et al., Safe radioisotope thermoelectric generators and heat sources for space applications, Journal of Nuclear Materials, 2008, 377(3), 506-521.

${ }^{8}$ Kelley, K. K. "The Specific Heats at Low Temperatures Of Crystalline Boric Oxide, Boron Carbide And Silicon Carbide". Journal of the American Chemical Society. 63 (1941) 1137-9.

${ }^{9}$ Kantor, K., P. B. Krasovitskaya, R. M. Kisil, O. M. Fiz. "Determining The Enthalpy And Specific Heat Of Beryllium In The Range 600-2200” Phys. Metals and Metallog. 10 (6) (1960) 42-4. Mcl-905/1, Ad-261792.

${ }^{10}$ Booker, J. Paine, R. M. Stonehouse, A. J. Wright. "Investigation Of Intermetallic Compounds For Very High Temperature Applications". Air Development Division (1961) 1-133. Wadd Tr 60-889, Ad 265625.

${ }^{11}$ Pankratz, L. B. K. K. Kelley. Thermodynamic Data for Magnesium Oxide U S Bur Mines. Report. 1-5 (1963); Bm-Ri-6295.

${ }^{12}$ Kandyba, K., V. V. Kantor, P. B. Krasovitskaya, R. M. Fomichev, E. N. Dokl "Determination Of Enthalpy And Thermal Capacity Of Beryllium Oxide In The Temperature Range From 1200 - 2820" Aec-Tr-4310. (1960) 1-4.

${ }^{13}$ Hedge, J. C., J. W. Kopec, C. Kostenko, J. I. Lang. Thermal Properties Of Refractory Alloys. Aeronautical Systems Division. (1963) 1-128; ( Asd-Tdr-63-597, Ad 424375 )

${ }^{14}$ Mattarolo, G., "Development and Modelling of a Thermophotovoltaic System," Dr.-Ing. Thesis, Electrical Engineering and Computer Science Dept., University of Kassel., Kassel, Germany, 2007.

${ }^{15}$ Howe, S. D., O’Brien, R. C., Ambrosi, R. M., Gross, B., Katalenich, J., Sailer, L, et al., "The Mars Hopper: An Impulse Driven, Long Range, Long-Lived Mobile Platform Utilizing In-Situ Martian Resources," Acta Astronautica, Vol. 69, Issues 1112, 2011, pp. 1050-1056.

${ }^{16}$ CD-adapco, Star-CCM+, CFD Software Package, Ver. 5.02, S. E. Eastgate Way, Bellevue, WA 98005.

${ }^{17}$ Autodesk, Inventor Professional 2012, CAD Software Package, McInnis Parkway, San Rafael, CA, 94903. 